\title{
Energy efficiency improvement of machine tools via peripheral devices management: an optimization-based control approach
}

\author{
Jenny L. Diaz C. and Carlos Ocampo-Martinez
}

\begin{abstract}
In this paper, a control scheme for both reducing the electric power consumption and minimizing power peaks during the operation of machine tools without affecting its throughput is proposed. The controller is designed to only manage peripheral devices without modifying the machining processes and the cycle time of the machine tool. Based on a test bench to emulate the energy consumption of a machine tool, a real data set is used to obtain models of electric power consumption by using data-driven model techniques such as subspace identification. Then, an optimization-based controller is designed considering both power consumption models and operating constraints of peripheral devices. The proposed controller is tested for both nominal and disturbed cases, i.e., with and without disturbances/uncertainties, achieving reductions up to $15 \%$ in the power peaks with respect to other control systems usually implemented for such systems. From this approach, both energy cost and economical penalties by overloads could be reduced and the energy efficiency of these machines can be improved.
\end{abstract}

\section{INTRODUCTION}

The depletion of fossil energy sources and the increasing energy prices have imposed new challenges to manufacturing industry, which accounts for $50 \%$ of energy consumed by the industrial sector [1]. From this fact, several strategies and methodologies that allow improving the energy efficiency of manufacturing systems by reducing energy costs and optimizing the use of resources have been proposed [2], [3].

A machine tool can be understood as an arrangement of different devices that work in a coordinated and sequential/parallel manner, and which can be classified into devices directly involved into the machining processes, and those that guarantee the operating conditions of these processes, namely as peripheral devices. During machine tool operation, energy costs are associated to total energy conversion in a fixed period and to economic penalties when the maximum contracted power is surpassed. This latter fact occurs due to the simultaneous activation of several devices, yielding in undesirable power peaks. Therefore, strategies that reduce the global energy conversion and avoid surpassing the nominal power, e.g., by selective on/off switching of the peripheral devices, could be useful to improve the energy efficiency of manufacturing systems without sacrificing their productivity.

Most of the strategies implemented so far to improve the energy efficiency of machine tools are focused on reduce the idle times and the total energy consumption by off-line optimization of the planning and scheduling of machining

All authors are with the Automatic Control Department, Universitat Politècnica de Catalunya, Institut de Robòtica i Informàtica Industrial (CSIC-UPC), Llorens i Artigas 4-6, 08028 Barcelona, Spain. e-mails: \{jdiaz, cocampo\}@iri.upc.edu processes [4], [5]. However, an alternative to solve this issue in real time is the design of control systems from which the machine devices can be managed considering the current energy consumption. In this regard, optimization-based control (OBC) techniques have had a great application due to their high customization level for defining control objectives, and the possibility of including operating constraints of both machining and peripheral devices into the controller design [6]-[8]. Nevertheless, one of the main concerns for designing control strategies based on optimization relies on the need for a suitable process model [3]. Thus, since the complexity of manufacturing systems, most of the models used for the design of control strategies are based on inputoutput correlations from data sets of energy consumption [2], [9], such as models obtained from subspace identification methods [10]. The counterpart, i.e., obtaining physicallybased models, requires the full knowledge of several physical dynamics and parameters, which are often hard to represent, compute or estimate.

This paper aims to propose an optimization-based control approach for energy consumption reduction and peak-power suppression by independently managing peripheral devices in order to reduce energy costs. The proposed controller is based on power consumption models determined by subspace identification (SI) methods, OBC techniques and the receding horizon philosophy to formulate an optimization problem that considers the operating constraints and relationships among the machining and peripheral devices. Thus, the main contribution of this work is to propose a control strategy that does not consider peripheral devices as isolated units but includes their energy consumption, their process dynamics, and their relations to machining process into the optimization problem. Hence, the time instants in which peripheral devices must be turned on to avoid surpassing the purchased nominal power and minimizing the global energy consumption are predicted and selected. The main result of the proposed approach is a control strategy that allows reducing energy costs without reducing the production of the machine. This approach is performed based on a real test bench that emulates the energy consumption of machining and peripheral devices of a machine tool.

The paper is organized as follows: Section II introduces the problem statement. Section III presents and discusses the proposed control approach, including the way to determine energy consumption models. Afterward, in Section IV, the considered case study and the way to determine the relations between the manufacturing and peripheral devices are presented and discussed. Next, obtained results for model 
identification and the proposed control strategy by simulation are shown in Section V. Finally, in Section VI, conclusions and future works based on the obtained results are drawn.

\section{Problem Statement}

A machine tool consists of a set of devices that can be classified as machining and peripheral devices. The former set is directly related to machining operations (cutting, milling, turning, etc.), while the latter set refers to devices that supply resources to machining devices and guarantee their correct operation. Thus, given the activation sequences of machining $\boldsymbol{\Lambda}_{\mathbf{M}}$ and peripheral devices $\boldsymbol{\Lambda}_{\mathbf{P}}$, an apparent power consumption $S$ is produced.

Based on the operation of machining devices, a periodic behavior characterizes a machine tool according to the total time required for manufacturing a piece, which corresponds to a machine cycle denoted as $T$. Due to different operations performed in a machine e.g., transport, rotational motions, axial motions, cutting, milling, etc., there exist operation stages of both high and low energy consumptions along $T$. Taking into account the stages of higher energy consumption, the peripheral devices should be properly managed such that their activation times do not match with the time instants/slots of higher consumption of the manufacturing operations, avoiding also (if possible) the simultaneous activation of peripheral devices.

Considering a fixed number of machining and peripheral devices related to a single ${ }^{1}$ machine, the activation sequences can be defined as follows:

$$
\begin{aligned}
\boldsymbol{\Lambda}_{\mathbf{M}}(k) & =\left\{u_{M_{1}}(k), u_{M_{2}}(k), \ldots, u_{M_{m}}(k)\right\}, \\
\boldsymbol{\Lambda}_{\mathbf{P}}(k) & =\left\{u_{P_{1}}(k), u_{P_{2}}(k), \ldots, u_{P_{n}}(k)\right\},
\end{aligned}
$$

being $k \in \mathbb{Z}_{\geq 0}$ the discrete-time index, $m=\left|\boldsymbol{\Lambda}_{\mathbf{M}}\right|$ and $n=\left|\boldsymbol{\Lambda}_{\mathbf{P}}\right|$ the number of machining and peripheral devices, respectively, while $u_{M_{j}}(k) \in\{0,1\}, l \in \mathcal{L} \triangleq\{1,2, \cdots, m\}$ and $u_{P_{j}}(k) \in\{0,1\}, j \in \mathcal{J} \triangleq\{1,2, \cdots, n\}$ are the activation times of the $l$-th machining device and the $j$-th peripheral device, respectively.

For the machining devices, the execution times $T_{M_{l}}$ are usually fixed and their operation is constrained into $T$. Therefore, $\sum_{l=1}^{m} T_{M_{l}}=T$ holds when only one machining device is turned on only once during $T$. On the other hand, since the peripheral devices might not show a periodic behavior, their operation is not constrained into $T$ and their execution times $T_{P_{j}}$ are not necessarily upper bounded by $T$. Given the periodicity of $\boldsymbol{\Lambda}_{\mathrm{M}}$, its apparent power consumption, namely $S_{M}$, can be considered as fixed and periodic (by the machine tool design). Consequently, both $u_{M_{l}}(k)$ and $T_{M_{l}}$ are given by the machining process and are known a priori. In this sense, Assumption 1 is established.

Assumption 1: The machining sequence $\boldsymbol{\Lambda}_{\mathbf{M}}$ is given and hence its associated apparent power consumption, denoted by $\bar{\beta} \triangleq \sum_{k=0}^{T} S_{M}\left(\boldsymbol{\Lambda}_{\mathbf{M}}(k)\right)$, when a periodic behavior in the machining process is considered.

\footnotetext{
${ }^{1}$ Without lost of generality, this notation represents the case of non-shared peripheral devices. The extension is straightforward.
}

On the other hand, the energy consumption from peripheral devices depends on the operational relations between machining and peripheral devices, which are needed to guarantee the operating conditions of the machining processes. Thereby, to select the optimal activation instants of the peripheral devices, mathematical expressions for energy consumption models and their operating constraints should be considered into the optimization problem to take into account the settling time of each element.

Thus, the control problem is to determine the optimal $\boldsymbol{\Lambda}_{\mathbf{P}}$ that minimizes both the global energy consumption $S$ and the power peaks that could exceed the nominal power purchased along a fixed period. Considering an operation time of length $T$, the first part of the control objective is defined as minimizing the area under the energy consumption profile, i.e.,

$$
J_{1}(k)=\bar{\beta}(k)+\sum_{k=1}^{k=T} S_{P}\left(k, \boldsymbol{\Lambda}_{\mathbf{P}}(k)\right),
$$

being $\bar{\beta}(k)$ the apparent power consumption produced by $\Lambda_{M}$, while for minimizing the highest peak, the second part of control objective is defined as

$$
J_{2}(k)=\|\mathbf{S}(k)\|_{\infty},
$$

with $S(k)=S_{P}\left(k, \boldsymbol{\Lambda}_{\mathbf{P}}(k)\right)+\beta(k)$, and $\mathbf{S}(k) \triangleq$ $\{S(k), \ldots S(k+T)\}$, for all $k \in \mathbb{Z}_{\geq 0}, S \in \mathbb{R}_{\geq 0}$.

Then, in order to achieve the control objectives, activation/deactivation of peripheral devices $j \in \mathcal{J}$ must be suitably managed subject to their operating constraints. Besides, to compute $S$, apparent power consumption models for each peripheral device are required, i.e., $S_{P_{j}}(k+1)=f_{j}\left(u_{P_{j}}(k)\right)$, where $f_{j}:\{0,1\} \mapsto \mathbb{R}_{\geq 0}$ is, in general, a nonlinear map in function of the individual input signal that activates/deactivates the $j$-th device. Hence, $S_{P}\left(k, \Lambda_{\mathbf{P}}(k)\right) \approx \sum_{j=1}^{n} S_{P_{j}}(k)$.

In addition, relationships among $\boldsymbol{\Lambda}_{\mathbf{M}}$ and $\boldsymbol{\Lambda}_{P}$ should be considered, since these determine the proper behaviors of the peripheral devices, e.g., the supply lubricant fluids from a central deposit through a pump, air from a compressor, chip transport, among others. These relationships, which could be of dynamic nature, can be defined as $q_{r}(k+1)=g_{r}\left(\nu, \boldsymbol{\Lambda}_{\mathbf{P}}(k)\right)$, for $r \in \mathcal{Q} \triangleq\{1,2, \cdots, Q\}$, where $Q$ is the number of existing relations $q$ and $\nu=$ $h\left(\boldsymbol{\Lambda}_{\mathbf{M}}\right) \in \mathbb{R}$ is the particular relation between $q_{r}$ and $\boldsymbol{\Lambda}_{\mathbf{M}}$. Besides, $h:\{0,1\} \times \mathbb{R} \mapsto \mathbb{R}$ and $g_{r}: \mathbb{R}^{m} \times\{0,1\}^{n} \mapsto \mathbb{R}$ are the maps that define such relations $q$. Notice that, in this case, $q$ is considered as some process variable of a particular peripheral device, which is directly related to $\Lambda_{M}$.

\section{Proposed Control Scheme}

For reducing energy consumption and avoiding power peaks during the machine tool operation, an optimizationbased controller is proposed considering both the energy consumption models and the operating constraints of peripheral devices into an optimization problem. Thus, the general idea is to use the receding horizon approach to anticipate either 
activation or deactivation of peripheral devices, taking into account their dynamics and the global energy consumption.

Considering a prediction horizon $H_{p}$, the decision of switching on or off the $j$-th device depends on the current value of $\beta(k)$, i.e., although the machining sequence is already given and hence its energy consumption, its consumption values discriminated along the time are important for making decisions regarding peripheral devices management. According to the defined control objectives in (2) and (3), the cost function could be defined as

$$
J(k)=\gamma_{1} \eta_{1} J_{1}(k)+\gamma_{2} \eta_{2} J_{2}(k),
$$

then, for $H_{p}$, the sequences ${ }^{2}$ for $J$ and $\boldsymbol{\Lambda}_{\mathbf{P}}$ are defined as

$$
\begin{aligned}
& \mathbf{J}(k) \triangleq\left\{J(k \mid k), \ldots J\left(k+H_{p}-1 \mid k\right)\right\}, \\
& \boldsymbol{\Gamma}(k) \triangleq\left\{\boldsymbol{\Lambda}_{\mathbf{P}}(k \mid k), \ldots, \boldsymbol{\Lambda}_{\mathbf{P}}\left(k+H_{p}-1 \mid k\right)\right\},
\end{aligned}
$$

with $\gamma_{1}$ and $\gamma_{2}$ being the weight coefficients for each objective, $\eta_{1}$ and $\eta_{2}$ the normalization values, respectively, $\mathbf{J}(k) \subseteq$ $\mathbb{R}^{H_{p}}, \boldsymbol{\Gamma}(k) \subseteq\{0,1\}^{n H_{p}}$, and the polytopic constraint

$$
\mathbb{Q}=\left\{q \in \mathbb{R}^{r} \mid q(k) \in\left[\underline{q}_{j}, \bar{q}_{j}\right] \forall k,\left\{\underline{q}_{j}, \bar{q}_{j}\right\} \in \mathbb{R}\right\} .
$$

being $\underline{q}_{j}$ and $\bar{q}_{j}$ the minimum and maximum values admissible for the $q$-relation of $j$-component. Next, the design of the proposed predictive-like controller is based on the following finite-time open-loop optimization problem:

$$
\min _{\boldsymbol{\Gamma}(\mathbf{k})} \mathbf{J}(k)
$$

subject to

$$
\begin{aligned}
q_{r}(k+i+1 \mid k) & =g_{r}\left(\nu, \boldsymbol{\Lambda}_{P}(k+i \mid k)\right), \\
S_{P_{j}}(k+i \mid k) & =f_{j}\left(\xi(k+i \mid k), u_{P_{j}}(k+i \mid k)\right), \\
u_{P_{j}}(k+i \mid k) & \in\{0,1\}, \\
q_{r}(k+i \mid k) & \in \mathbb{Q},
\end{aligned}
$$

being $\xi(k+i \mid k)$ the states of the energy consumption model, $i \in\left[0, H_{P}-1\right], j \in \mathcal{J}, r \in \mathcal{Q}$, and $r=j$ if there is only one $q$-relation for each $j$-component.

Assuming that the problem (7) is feasible, i.e., $\boldsymbol{\Gamma}(k) \neq \emptyset$, there will be an optimal solution for the activation sequence of peripheral devices defined by

$$
\boldsymbol{\Gamma}^{*}(k) \triangleq\left\{\boldsymbol{\Lambda}_{\mathbf{P}}{ }^{*}(k \mid k), \ldots, \boldsymbol{\Lambda}_{\mathbf{P}}{ }^{*}\left(k+H_{P}-1 \mid k\right)\right\},
$$

and then, according to the receding horizon philosophy [11], [12], $\boldsymbol{\Lambda}_{\mathbf{P}}{ }^{*}(k \mid k) \in\{0,1\}^{n}$ is applied to the system discarding the rest of the optimal sequence from $(k+1) \mid k$ to $\left(k+H_{p}-1\right) \mid k$, while the whole process is repeated for the next time instant $k \in \mathbb{Z}_{\geq 0}$ after measuring/estimating the proper information from the plant to be used as the update for the energy consumption models considered in (7c). According to (7), suitable expressions for maps $f_{j}$ and $g_{r}$ should be proposed based on the real operation of the peripheral devices in the considered machine tool.

\footnotetext{
${ }^{2}$ Here, $z(k+i \mid k)$ denotes the prediction over $H_{p}$ of the variable $z$ at time instant $k+i$ performed at $k$.
}

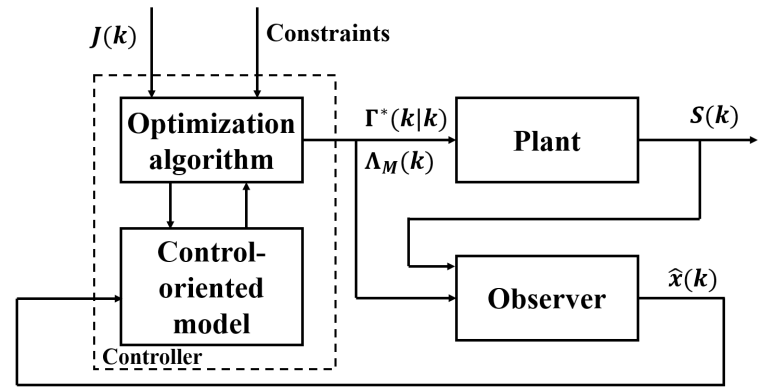

Fig. 1: Control scheme of energy consumption in a machine tool.

\section{A. Subspace identification}

Given the possible nonlinear nature of maps $f_{j}$, this paper proposes the identification of input-output models based on the SI methods. From a proper set of input-output data i.e., activation sequences $\boldsymbol{\Lambda}_{\mathbf{P}}$ and apparent power $S_{P}$, SI methods allow the identification of matrices of a state space realization for Linear Time-Invariant (LTI) systems. The main advantage of these methods is that the state-space realizations are quite convenient for estimation, control, and prediction tasks.

Thus, for a given set of input-output measurements of length $d$, the SI problem consists in estimating both the system order $N$ and the model matrices [13]. Then, to determine the model matrices and $N$, this paper focuses on the Numerical algorithms For Subspace IDentification (N4SID) [14] because of the great application and its implementation in software. The N4SID algorithms first estimate a state $\hat{x} \in \mathbb{R}$ from the projection of input-output data, and then, system matrices are determined based on this estimated state sequence. A detailed review about the different SI algorithms and their implementation can be found in [10], [13]-[15].

\section{B. Control scheme based on optimization}

According to the optimization problem formulated in (7) and considering the receding horizon approach, a predictivelike controller is proposed. Considering the power consumption models, $q$-relations, and the operating ranges of peripheral devices, the proposed control scheme to improve energy efficiency of a machine tool is depicted in Figure 1.

In the proposed closed-loop control scheme, the optimization problem in (7) is solved into a optimizer module. Afterward, once an optimal $\boldsymbol{\Gamma}^{*}(k)$ is determined, only the first component $\boldsymbol{\Lambda}_{\mathbf{P}}{ }^{*}(k \mid k) \in\{0,1\}^{n}$ is sent to both the plant and the model of the observer module for feeding back the optimization algorithm. Next, the current state obtained from the observer module and measured outputs are fed back to the optimizer module at each $k$. Since the system is composed of the individual power consumption models for both the machining and peripheral devices, the total output $S$ can be defined as the sum of $S_{M}$ and $S_{P}$, previous to the design of the observer module. Thus, a total power consumption model is defined by extending the model matrices as follows:

$$
A_{e x}=\left[\begin{array}{cccc}
A_{M} & 0 & \cdots & 0 \\
0 & A_{P_{1}} & \cdots & 0 \\
\vdots & \vdots & \ddots & \vdots \\
0 & 0 & 0 & A_{P_{j}}
\end{array}\right] \text {, }
$$




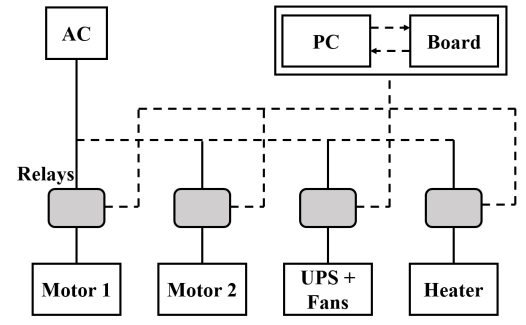

Fig. 2: Diagram of test bench of a machine tool emulator.

and, similarly, for $B_{e x}, C_{e x}$, and $D_{e x}$. Considering $u(k)=\left[u_{M}(k), u_{P_{1}}(k), \ldots, u_{P_{j}}(k)\right]$ and $x(k)=$ $\left[x_{M}(k), x_{P_{1}}(k), \ldots, x_{P_{j}}(k)\right]$ the extended input and state vectors, respectively, a Kalman filter to estimate the states of both machining and peripheral devices models from the overall system output $S$ is designed as follows:

$$
\begin{aligned}
\hat{x}(k+1) & =A_{e x} \hat{x}(k)+B_{e x} U(k)+L(S(k)-\hat{S}(k)), \\
\hat{S}(k) & =C_{e x} \hat{X}(k)+D_{e x} u(k),
\end{aligned}
$$

being $\hat{x}$ and $\hat{S}$ the estimation vectors of state and output, respectively, and $L$ the observer gain matrix. Then, given the nature of the optimization problem, which is a mixed integer linear programming problem, the optimizer module in Figure 1 uses optimization software IBM ILOG CPLEX Optimization Studio integrated to YALMIP toolbox [16] to find the optimal input $\boldsymbol{\Gamma}^{*}(k)$ that minimizes both the global energy consumption and the infinity norm along $H_{p}$.

\section{CAse Study}

A test bench has been built to emulate the energy consumption behavior of a machine tool and its peripheral devices with the aim to extract data. Due to the nature of devices in a machine, different types of loads were considered to emulate the real power consumption of both machining and peripheral devices. The test bench is composed of a three-phase star connection motor, a three-phase delta connection motor, a heater, a uninterrupted power supply (UPS) device, and one data acquisition module with a sampling rate up to $250 \mu \mathrm{s}$. In addition, a set of electronic devices, a PC, and a development board are included to control relays, which allows either activation or deactivation of the testbench components according to an activation sequence sent. Thus, through the acquisition device, data are taken and sent to both board and PC, which receives the power signal $S$ [VA] for each phase according to the activation sequence sent to the test bench. An diagram of the test bench with the connected loads is shown in Figure 2.

Based on the available components in the test bench, and due to the periodic behavior of a machine tool, the test-bench components were classified as peripheral and machining devices. In this sense, a heater and a UPS were used to construct the machining sequence while the available motors will be considered as the peripheral devices. This selection is made since the energy consumption of both the heater and the UPS can be modulated to emulate the machining sequence, whereas motors were selected as peripheral devices since they usually produce instantaneous peaks when activated.
TABLE I: Simulation parameters for peripheral devices.

\begin{tabular}{cc|c|c|c|c}
\hline $\mathbf{j}$ & $\alpha_{j}\left(\boldsymbol{\Lambda}_{M}\right)$ & $\eta_{j}\left(\boldsymbol{\Lambda}_{M}\right)$ & $\underline{q}_{j}$ & $\bar{q}_{j}$ & $q_{j}(0)$ \\
\hline $\mathbf{P}_{\mathbf{1}}$ & $-3[\mathrm{ud} / \mathrm{s}]$ & $5[\mathrm{ud} / \mathrm{s}]$ & 50 & 150 & 55 \\
\hline $\mathbf{P}_{\mathbf{2}}$ & $-5[\mathrm{ud} / \mathrm{s}]$ & $7[\mathrm{ud} / \mathrm{s}]$ & 60 & 180 & 65 \\
\hline
\end{tabular}

According to the previous discussion, a machining sequence $\Lambda_{\mathrm{M}}$ is created with $T=28 \mathrm{~s}$, which is constant along the time. This latter fact does not hold for peripheral devices, which will be activated depending on both the global $S$ and the $q$-relations between each device and $\boldsymbol{\Lambda}_{\mathbf{M}}$. Then, different tests were performed and a sampling time of $\tau_{s}=0.01 \mathrm{~s}$ was selected based on a trade-off between the temporal resolution of the signals and a suitable computational time for running the proposed designs. In Figures $3 a$ and $3 b$, the energy consumption profiles of peripheral devices and $\Lambda_{M}$ (dotted line) for one of the machine phases $\left(S_{B}\right)$ are presented, as it is developed in most of proposed works [17], [18].

\section{A. q-relations}

For this case study, it is considered that some information of the process variables about a resource consumed by the machine and provided by the peripheral devices is available. Thus, based on a given process variable, a relation regarding the amount of resources consumed along $T$ and an incremental value when the peripheral device is turned on are fixed. From this fact, $g_{r}\left(\nu, \boldsymbol{\Lambda}_{\mathbf{P}}(k)\right)$ is defined as a linear relationship between $\Lambda_{M}$ and $\Lambda_{P}$ based on the dynamics of resources consumed by the machine. In this sense, for both delta $\left(P_{1}\right)$ and star connections $\left(P_{2}\right)$ of the motor, a linear dynamic model is established as follows:

$$
q_{j}(k+1)=q_{j}(k)+\alpha_{j}\left(\boldsymbol{\Lambda}_{M}\right)+\eta_{j}\left(\boldsymbol{\Lambda}_{M}\right) u_{P_{j}}(k),
$$

being $j=1,2$ corresponding to one relation for each peripheral devices, and $\eta_{j}\left(\boldsymbol{\Lambda}_{M}\right)$ and $\alpha_{j}\left(\boldsymbol{\Lambda}_{M}\right)$ the increase and decrease factors of the $j$-component at each $k$, respectively. Therefore, since the machine tool requires the resources delivered from peripheral devices for its suitable operation, $q_{j}(k)$ must belong to a given operating range. In Table I, the values of $\alpha_{j}\left(\boldsymbol{\Lambda}_{M}\right), \eta_{j}\left(\boldsymbol{\Lambda}_{M}\right)$ and the operating ranges of $q_{j}(k)$ are presented for each $j$-component considered.

It should be noted that, although in the test bench there are three phases available to connect devices, in this work, all components have been connected in only one phase due to the small number of available components. However, the analysis proposed for one phase can be properly extended for handling devices connected to all phases [17], [18].

\section{Simulation Results}

\section{A. Model identification}

In this case, three models of energy consumption were identified. To this end, different sequences of $\boldsymbol{\Lambda}_{\mathbf{P}}$ and $\boldsymbol{\Lambda}_{M}$ were tested to obtain the corresponding outputs $S_{P}$ [VA] and $S_{M}$ [VA]. Afterward, energy consumption models were identified by using the command $\mathrm{n} 4 \mathrm{sid}$ of the System Identification Toolbox ${ }^{\mathrm{TM}}$ provided by Matlab $\mathrm{R}$.

Based on the obtained data sets, different values of $N$ to identify the matrices $A, B, C$, and $D$, which allow the 
higher fitting degree between both real and modeled outputs, were tested into System Identification Toolbox ${ }^{\mathrm{TM}}$. From the obtained results, for each one of models identified, the order selected was $N=3$ since it represents the lowest values from which a high fitting degree between measurements and modeled outputs is obtained. In Figures $3 a$ and $3 b$, the validations of obtained models for both peripheral devices and machining process are shown, respectively.

The fitting percentages of each model output with respect to the available real data have been $97.61 \%$ for $P_{1}, 99.07 \%$ for $P_{2}$ and $99.86 \%$ for the machining sequence. From these results, it is possible to observe that identified models are able to represent the dynamic behavior of both peripheral devices and machining sequence with enough accuracy.

\section{B. Weight and Normalization coefficients}

Based on (4), the normalization and weight coefficients must be determined according to the admissible values for each objective considered. For the case of $\eta_{1}$ and $\eta_{2}$, the possible minimum and maximum values to bring all values in the range $[0,1]$ are taken based on the considered case study. Regarding $J_{1}$, the minimum value corresponds to the area under the energy consumption profile for $\Lambda_{M}$, while the maximum value is taken as the global energy consumption when both peripheral devices are turned on along $H_{p}$. For the case of $J_{2}$, the minimum and maximum values correspond to the higher peak of $\Lambda_{\mathrm{M}}$ and the sum of $S$ when both peripheral devices are turned on at the same instant in which the higher peak of $\boldsymbol{\Lambda}_{\mathrm{M}}$ occurs, respectively.

Then, for determining values of $\gamma_{1}$ and $\gamma_{2}$ a trial-anderror tuning procedure is used to find the trade-off between the proposed control objectives. For this purpose, variations of 0.05 for both $\gamma_{1}$ and $\gamma_{2}$ were evaluated and the values of $J_{1}$ and $J_{2}$ were analyzed in order to select the suitable values of $\gamma_{1}$ and $\gamma_{2}$. According to the tuning performed, $\gamma_{1}=0.9$ and $\gamma_{2}=0.1$ were selected as the best trade-off between the proposed control objectives taking into account that in this proposal $J_{1}$ is prioritized over $J_{2}$.

\section{Prediction Horizon}

Given the periodic behavior of machine tools, values of $H_{p}$ equal to $T,\left(T+\frac{T}{2}\right)$, and $2 T$ were tested to determine the more suitable length of $H_{p}$. The values of $J_{1}$ and $J_{2}$ were compared among them and, the case in which both $J_{1}$ and $J_{2}$ are significantly reduced was selected as the length of $H_{p}$. The obtained results are shown in Figure 3c.

The results in Figure 3c shows the energy consumption profile for different values of $H_{p}$ during a total simulation time of $T_{s}=3 T$. From these results, it is possible to appreciate that, for large values of $H_{p}$ the height of peaks is increased, while for $H_{p}=T$ the height of peaks is lower and the energy consumption profile is smoother. In Table II, the values of $J_{1}, J_{2}$ and the computing time $t_{c}$ are presented. Based on these results, $H_{p}=T$ is selected to evaluate the proposed control strategy since that value reaches a suitable trade-off between both objectives, showing the lowest computational time. Thus, if it is considered to apply the whole optimal sequence to the system instead of
TABLE II: Fitting percentages of real and modeled outputs.

\begin{tabular}{cccc}
\hline Objective & $H_{P}=T$ & $H_{P}=T+\frac{T}{2}$ & $H_{P}=2 T$ \\
\hline$J_{1}[\mathrm{VA}]$ & $3.3416 \times 10^{4}$ & $3.4418 \times 10^{4}$ & $3.4050 \times 10^{4}$ \\
\hline$J_{2}[\mathrm{VA}]$ & 585.69 & 605.76 & 624.79 \\
\hline$t_{c}[\mathrm{~s}]$ & 1.74 & 4.81 & 24.61 \\
\hline
\end{tabular}

the first component of $u_{P_{j}}^{*}, t_{c}$ is significantly smaller than a machine cycle $T=28 \mathrm{~s}$. In addition, from $H_{p}=T$ the whole machine cycle is considered into the optimization problem, which would imply to make a better decision from the input selection perspective.

It should be noted that although the sampling time was fixed as $\tau_{s}=0.01 \mathrm{~s}$, the proposed controller is executed to find optimal values of $u_{P_{j}}$ at each second and keeping this value until the next one. This way of implementing the controller is considered given the amount of data to be processed and the requirements of computing time for further real-time implementations.

\section{Energy efficiency control}

Based on the proposed control scheme and values of $\eta_{1}, \gamma_{1}, \eta_{2}, \gamma_{2}$ and $H_{p}$ defined in previous sections, simulation results are presented below. According to parameters in Table I for relations $q_{j}(k)$, all simulations were performed in Matlab $®$ considering a total simulation time $T_{S}=8 T$, while the designed Kalman filter was validated using real data from the test bench showing satisfactory results.

Taking into account the $q$-relations and operating constraints such as safety time $t_{s}$ for keeping turn on/off some device, simulation results for the proposed control strategy are presented in Figures 4a, 4b, and 4c. In this case, two different simulation horizons $H_{S}$ were compared since the periodic behavior of these machines. In the first one, the conventional MPC strategy, in which only the first component is applied to the system $\left(H_{S}=1 \mathrm{~s}\right)$ is considered, while in the second one, the whole optimal sequence for all $H_{p}$ is applied to system $\left(H_{S}=T\right)$.

All simulations were performed considering the same safety time for all the peripheral devices, $t_{s}=5 \mathrm{~s}$. Safety constraints are required to avoid damages due to high on/off frequencies, which affect the inertia of these devices. Therefore, at each time when some device is turned on/off, it must keep equal during at least $5 \mathrm{~s}$. In Figures $4 \mathrm{a}, 4 \mathrm{~b}$ and $4 \mathrm{c}$, the proposed predictive-like control (Energy-Efficiency Control, EEC) is compared to other basic control (BC) that only considers the bounded values of $\bar{q}_{j}$ and $\underline{q}_{j}$ for selecting the activation instants of peripheral devices.

In Figure $4 \mathrm{a}$, it is possible to see that, according to the proposed control objectives, the height of power peaks is minimized with respect of $\mathrm{BC}$, and therefore, economic penalties for surpassing the nominal power can be avoided. This reduction in energy costs is related to the delay in the activation of peripheral devices in order to avoid their simultaneous activation. According to the reported results, the highest peaks registered using the EEC were $S_{\max }=623.2 \mathrm{VA}$ and $S_{\max }=585.7 \mathrm{VA}$ using $H_{S}=1 \mathrm{~s}$ and $H_{S}=T$, respectively, while for the BC the highest peak was $S_{\max }=661.7 \mathrm{VA}$. In this regard, improvements up to 


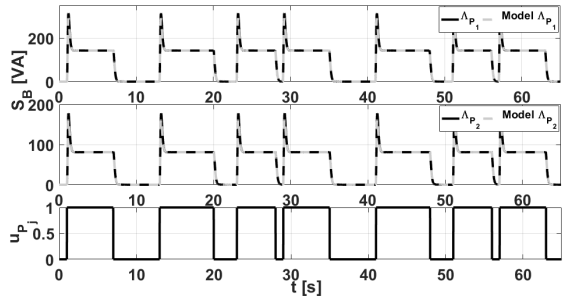

(a)

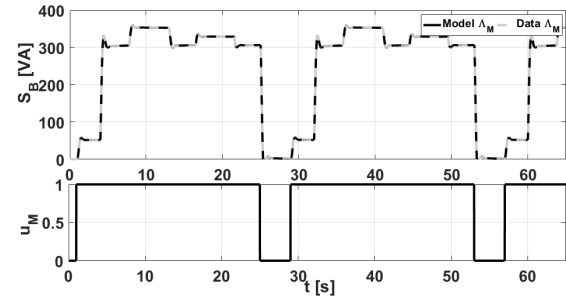

(b)

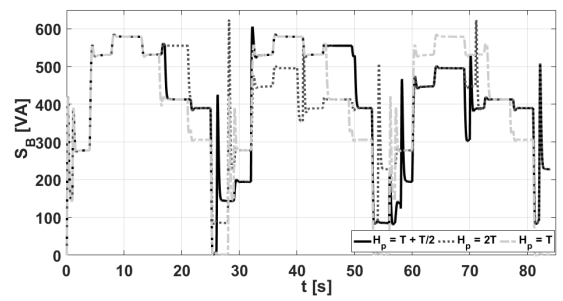

(c)

Fig. 3: Different assessments. (a) Input signal and model validation of peripheral devices. (b) Input signal and model validation of machining sequence (MS). (c) Comparative of different values of $H_{p}$ for an initial optimization.

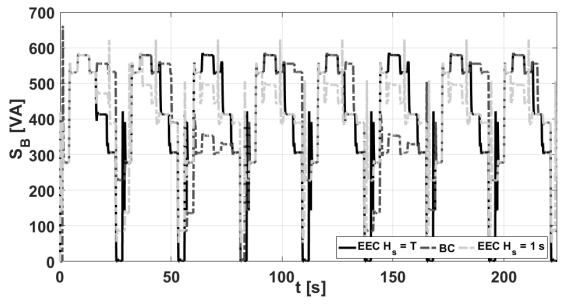

(a)

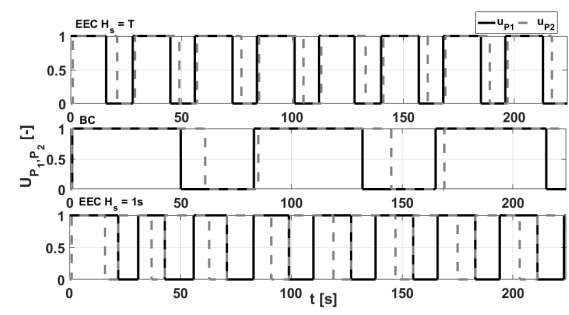

(b)

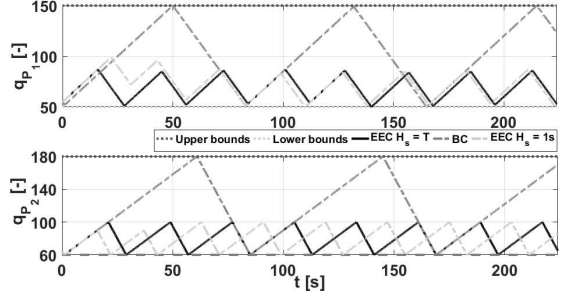

(c)

Fig. 4: (a) Total apparent power consumption during the machine operation. (b) Input signals of the peripheral devices for the different control strategies tested. (c) Dynamic of $q_{r}$ for each one of considered peripheral devices.

$6 \%$ were achieved with respect to $J_{2}$ without affecting the machine production. This fact is given since the machining sequence is fixed and, therefore, the time to process a piece is always the same.

According to Figure $4 \mathrm{~b}$, the signals $u_{P_{1}}$ and $u_{P_{2}}$ obtained from the EEC never turned on at the same time and, trying to turn off the peripheral device of higher consumption $\left(P_{1}\right)$ always that it is not required, and therefore, this device has a switching frequency greater than results obtained with the BC. A similar case can be appreciated for device $P_{2}$ in which the switching frequency is increased regarding the BC. Thus, the EEC tries to keep the peripheral devices near the minimum values admissible for $q_{j}$ and then, turning them off when they are far away from $\underline{q}_{j}$ to avoid unnecessary energy consumption. Although the proposed strategy increases the switching frequency for both devices, the safety constraints and the operating relations represented here for $q_{1}$ and $q_{2}$ are always satisfied as shown in Figure 4c.

In order to evaluate the performance of the proposed control strategy, the key performance index named Specific Energy Consumption (SEC), which expresses the ratio of total energy consumption to the effective output of a machining process, is calculated according to [19]. The values of both SEC and $J_{1}$ for the tested control approaches are presented in Table III. Regarding $J_{1}$, global energy consumption is not significantly reduced since the energy consumption behavior of the peripheral devices is not modified and, according to Figure $4 \mathrm{c}$, the operational cycle of peripheral devices $T_{P}$ with the $\mathrm{BC}$ strategy is greater than the considered prediction horizon $H_{p}=T$. This latter fact produces that the mentioned comparison will not be both realistic and fair since one of the proposed strategies could be favored
TABLE III: Assessment of SEC and $J_{1}$ values.

\begin{tabular}{cccc}
\hline Index & EEC $\left(H_{s}=1 \mathrm{~s}\right)$ & EEC $\left(H_{s}=T\right)$ & BC \\
\hline $\mathbf{J}_{\mathbf{1}}[\mathrm{VA}]$ & $8.9491 \times 10^{4}$ & $8.9210 \times 10^{4}$ & $9.1769 \times 10^{4}$ \\
\hline SEC $[\mathrm{VA}]$ & $1.1186 \times 10^{4}$ & $1.1151 \times 10^{4}$ & $1.1471 \times 10^{4}$ \\
\hline
\end{tabular}

TABLE IV: SEC and $J_{i}$ values for both ECC and BC tested.

\begin{tabular}{cccc}
\hline Index & $\mathbf{J}_{\mathbf{1}}[\mathrm{VA}]$ & $\mathbf{J}_{\mathbf{2}}[\mathrm{VA}]$ & $\mathbf{S E C}[\mathrm{VA}]$ \\
\hline $\mathbf{E E C}\left(H_{s}=T\right)$ & $7.488 \times 10^{4}$ & 728.62 & $1.248 \times 10^{4}$ \\
\hline $\mathbf{B C}$ & $8.032 \times 10^{4}$ & 860.05 & $1.347 \times 10^{4}$ \\
\hline
\end{tabular}

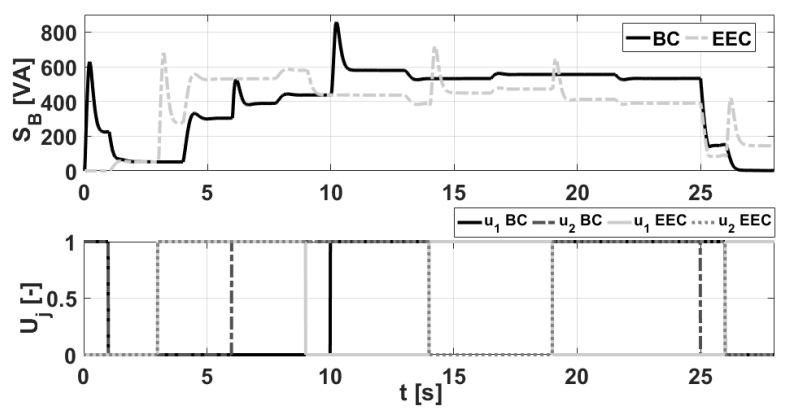

Fig. 5: Comparative between EEC and $\mathrm{BC}$ using $H_{p}=T_{P}$. depending on the time period tested. However, as a way of comparing the performance of EEC and BC approaches under the same conditions, in Figure 5 a hypothetical case in which $H_{p}=T_{P}$ is presented. From these results, it is possible to see that, when the optimization is performed for the same length of $T_{P}$, better results can be obtained with improvements around $6.3 \%$ and $22 \%$ for $J_{1}$ and $J_{2}$, respectively.

\section{E. Disturbances handling}

In order to evaluate the performance of the proposed control strategy in a more complex situation, a case in 


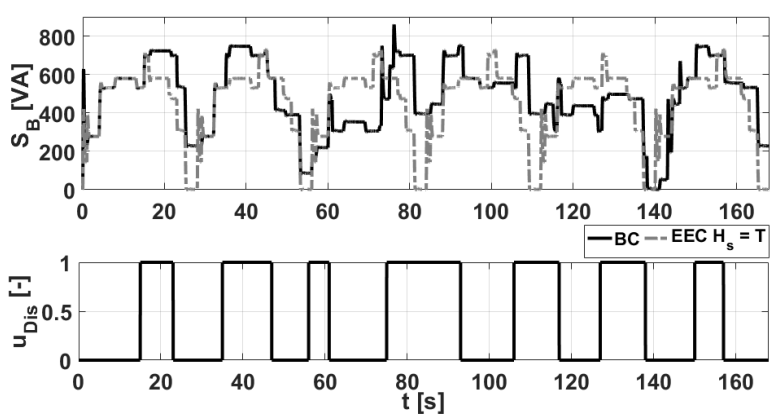

Fig. 6: (a) Total power consumption. (b) Unknown input.

which one load not consider into the optimization problem is activated for some time intervals is analyzed and compared with BC. In Figure 6, the activation signal of an unexpected load and the apparent power resulting from the application of both EEC and BC are presented. The obtained results for $J_{1}, J_{2}$ and SEC are summarized in Table IV.

According to Table IV, a reduction of $7.4 \%$ per machine cycle in the global energy consumption could be achieved using the EEC when disturbances are considered. This fact is given due to the inherent robustness shown by the optimization-based controllers (as the predictive ones) and their non-static control law philosophy. On the other hand, regarding $J_{2}$, improvements of $15 \%$ are reached when disturbances affect the system since the proposed control is able to manage the activation instants of the peripheral devices taking into account both the energy consumption of the machining sequence and the difference between the measured and the estimated output. In this sense, although for the nominal case energy cost reductions are achieved, a more significant effect of the proposed control strategy is appreciated when non-considered scenarios in the control design appear, as occurs in a real industry for which it is not possible to handle all the factors that potentially affect the system.

\section{CONCLUSIONS AND FUture WORKS}

Significant improvements in reducing the power peaks magnitude can be achieved with the proposed control strategy without compromise the machine operation and its production. Regarding the global energy consumption, significant reductions cannot be achieved because of the energy consumption behavior of peripheral devices is not modified or modulate. Thus, including peripheral devices with different power consumption levels could be an interesting work in order to improve the system flexibility. Besides, a sensitivity analysis both of design and simulation parameters should be performed in order to guarantee the proper operation of the control scheme under different operational conditions.

Besides, more realistic relationships between machining and peripheral devices can be included and analyzed in order to add more realism and complexity to the ongoing work. The dynamics of the typical peripheral devices (and not only their energy consumption behavior) could be considered into the optimization problem by using proper modeling techniques. Finally, disturbances as damage in the operation of peripheral devices could also be considered with the aim to evaluate the performance and robustness of the proposed strategy.

\section{ACKNOWLEDGMENT}

This work has been partially funded by the Spanish projects DEOCS (ref. MINECO DPI2016-76493) and the Spanish State Research Agency through the María de Maeztu Seal of Excellence to IRI (MDM-2016-0656). Authors would like to thank Ikergune (Exte-Tar Group) and the project IKERCON (ref. C10683) for their scientific support in this work.

\section{REFERENCES}

[1] IEA, "International energy agency: World energy statistics 2017," http://www.iea.org/statistics/, 2018.

[2] L. Zhou, J. Li, F. Li, Q. Meng, J. Li, and X. Xu, "Energy consumption model and energy efficiency of machine tools: a comprehensive literature review," Journal of Cleaner Production, vol. 112, no. Part 5, pp. $3721-3734,2016$.

[3] J. R. Duflou, J. W. Sutherland, D. Dornfeld, C. Herrmann, J. Jeswiet, S. Kara, M. Hauschild, and K. Kellens, "Towards energy and resource efficient manufacturing: A processes and systems approach," CIRP Annals - Manufacturing Technology, vol. 61, no. 2, pp. 587-609, 2012.

[4] F. Shrouf, J. Ordieres-Meré, A. García-Sánchez, and M. OrtegaMier, "Optimizing the production scheduling of a single machine to minimize total energy consumption costs," Journal of Cleaner Production, vol. 67, pp. 197-207, 2014.

[5] Z. Zhang, R. Tang, T. Peng, L. Tao, and S. Jia, "A method for minimizing the energy consumption of machining system: integration of process planning and scheduling," Journal of Cleaner Production, vol. 137, pp. 1647-1662, 2016.

[6] S. Ubach, J. Diaz, C. Ocampo-Martinez, and M. Antunez, "Peak shaving through closed-loop optimization applied to machine tools with periodic behaviour," in 2017 IEEE 3rd Colombian Conference on Automatic Control (CCAC), 2017, pp. 1-7.

[7] Y. Halevi, E. Carpanzano, G. Montalbano, and Y. Koren, "Minimum energy control of redundant actuation machine tools," CIRP Annals, vol. 60, no. 1, pp. 433-436, 2011.

[8] N. Frigerio and A. Matta, "Energy efficient control strategy for machine tools with stochastic arrivals and time dependent warm-up,' Procedia CIRP, vol. 15, pp. 56-61, 2014.

[9] R. Bhinge, J. Park, K. H. Law, D. A. Dornfeld, M. Helu, and S. Rachuri, "Toward a generalized energy prediction model for machine tools," Journal of Manufacturing Science and Engineering, vol. 139, no. 4, pp. 1-12, 2016

[10] S. J. Qin, "An overview of subspace identification," Computers \& Chemical Engineering, vol. 30, no. 10, pp. 1502 - 1513, 2006.

[11] J. Maciejowski, Predictive Control with Constraints. Great Britain: Prentice Hall, 2002.

[12] J. Rawlings and D. Mayne, Model Predictive Control: Theory and Design. Madison, WI (USA): Nob Hill Publishing, 2009.

[13] P. Overschee and B. De Moor, Subspace Identification for Linear Systems : Theory - Implementation - Applications. Boston, MA: Springer US, 1996.

[14] M. Verhaegen and A. Hansson, "N2sid: Nuclear norm subspace identification of innovation models," Automatica, vol. 72, pp. 57 63, 2016.

[15] B. De Moor, P. Van Overschee, and W. Favoreel, Algorithms for Subspace State-Space System Identification: An Overview. Boston, MA: Birkhäuser Boston, 1999, pp. 247-311.

[16] J. Löfberg, "Yalmip : A toolbox for modeling and optimization in matlab," in In Proceedings of the CACSD Conference, Taipei, Taiwan, 2004.

[17] W. Lee, S. H.K., J. Park, and B. Min, "Simulation-based machining condition optimization for machine tool energy consumption reduction," Journal of Cleaner Production, vol. 150, pp. 352-360, 2017.

[18] R. Popp, C. Liebl, and M. Zaeh, "Evaluation of the energy flexible operation of machine tool components," Procedia CIRP, vol. 63, pp. 76-81, 2017.

[19] L. Li, C. Li, Y. Tang, and Q. Yi, "Influence factors and operational strategies for energy efficiency improvement of CNC machining," Journal of Cleaner Production, vol. 161, pp. 220-238, 2017. 